\title{
A NEW VARIANT OF TEMPORARY TEMPERATURE FIELD CORRECTION METHOD
}

\author{
Bohdan Mochnacki ${ }^{1,3}$, Ewa Majchrzak ${ }^{2}$, Romuald Szopa ${ }^{1}$ \\ ${ }^{1}$ Czestochowa University of Technology, Poland \\ ${ }^{2}$ Silesian University of Technology, Poland \\ ${ }^{3}$ Higher School of Labour Safety Management, Katowice, Poland \\ bohdan.mochnacki@im.pcz.pl,ewa.majchrzak@polsl.pl,romuald.szopa@word.katowice.pl
}

\begin{abstract}
The paper presents the possibilities of numerical solution of the non-linear boundary-initial problem described by the Fourier equation. In particular, the equation containing the temperature-dependent thermophysical parameters (volumetric specific heat and thermal conductivity) is considered. The problem presented in this paper is connected with the artificial linearization of the task discussed (at the stage of numerical computations), in other words, the new numerical procedure which allows one to remodel the solution obtained for linear problem at the time level $t+\Delta t$ to the other solution corresponding to nonlinear one. The procedure discussed can be a very effective supplement for different variants of the boundary element method which, as a rule, requires a linear form of the energy equation. In the final part the examples of numerical simulations and the conclusions can be found.
\end{abstract}

\section{Introduction}

The following energy equation is considered:

$$
x \in \Omega: c(T) \frac{\partial T(x, t)}{\partial t}=\nabla[\lambda(T) \nabla T(x, t)]
$$

where: $\lambda(T)$ is the thermal conductivity, $c(T)$ is the volumetric specific heat, $T=T(x, t), x, t$ denote temperature, spatial co-ordinates and time, respectively. The equation (1) is supplemented by the boundary conditions in a general form

$$
x \in \Gamma: \quad \Phi\left[T(x, t), \frac{\partial T(x, t)}{\partial n}\right]=0
$$

where $\partial T / \partial n$ denotes the normal derivative. The initial condition

$$
t \in 0: \quad T(0, t)=T_{0}(x)
$$

is also given 
Let us assume that we know the temporary solution (corresponding to time $t+\Delta t$ ) of the linear problem

$$
x \in \Omega \quad: c_{B} \frac{\partial T_{B}(x, t)}{\partial t}=\lambda_{B} \nabla^{2} T_{B}(x, t)
$$

where $\lambda_{B}$ and $c_{B}$ are the certain constant 'basic' parameters. The boundary and initial conditions of the both problems are the same. The question for which we will try to find the answer is how to remodel the solution of problem (4) to the solution of problem (1).

At present in the literature one can find the several procedures which allow one to transform the temporary solution of linear problem (for transition from time $t$ to time $t+\Delta t$ ) to the solution corresponding to the non-linear one. In this place one should specify:

- the artificial heat source method presented by Majchrzak and Mochnacki among others in $[1,4,5]$,

- the alternating phase truncation method [6] generalized by Mochnacki and Kapusta (e.g. [7, 8]),

- the temporary temperature field correction method (proposed by Hong, Umeda and Kimura) and next developed by Majchrzak, Mochnacki [3, 4] and Szopa [9],

- the generalization of temporary temperature field correction method presented in this paper.

\section{Temporary temperature field correction method}

The numerical procedure being the 'prototype' of TFCM appeared in the scientific environment of people working in the scope of thermal theory of foundry processes many years ago (temperature recovery method [1]). The approximate solution of the well known Stefan problem (e.g. solidification of pure metals) has been obtained on the basis of a solution concerning the cooling process in homogeneous domain, but when the local temperature decreases below the solidification point $T^{*}$, then at the node considered the value of $T^{*}$ should be assumed, while the latent heat $L$, this means $A_{2}-A_{1}$, should be decreased about change of local enthalpy resulting from the cooling process proceeding at this point during time interval $\Delta t$ (Fig. 1).

In 1984 Hong, Umeda and Kimura [2] proposed to apply the similar algorithm for the case of alloys solidification (the course of enthalpy for such material is shown in Figure 2, at the same time the values of successive volumetric specific heats are assumed to be constant).

The computations are realized for a conventionally homogeneous domain (e.g. molten metal). When the local temperature decreases below the value corre- 
sponding to the beginning of solidification process then the nodal temperature obtained is corrected on the basis of energy balance resulting from the change of local enthalpy. The concept of temperature field correction is shown in Figure 2. In this place it should be pointed out that the creators of procedure discussed have not noticed that the proposed approach is correct only under the assumption of constant value of thermal conductivity in whole domain $\Omega$ (see: $[3,4,9]$ ).

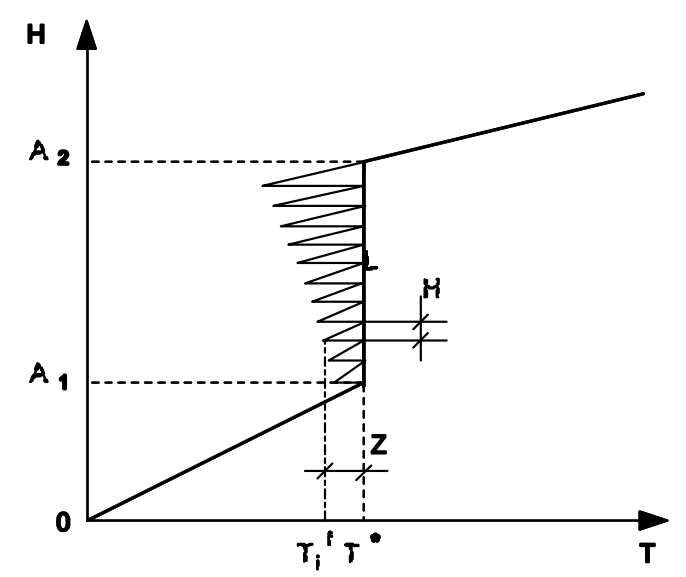

Fig.1. Temperature recovery method

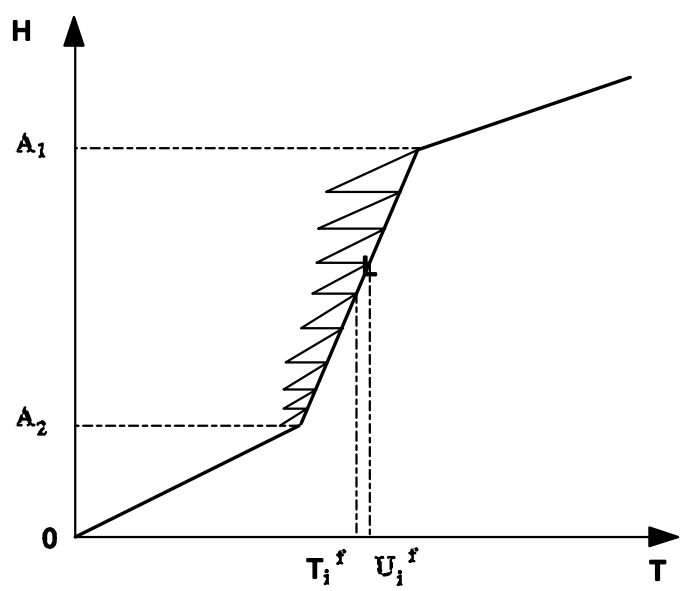

Fig. 2. Procedure proposed by Hong, Umeda and Kimura

The generalized version of TFCM will be presented sufficiently in detail, because the 'better' version of the method which will be presented in the next chapter basis on similar assumptions and formulas.

Let us assume the thermal conductivity of domain $\Omega$ is a constant value, while the volumetric specific heat is approximated by the piecewise constant function. In other words, the following energy equation is considered: 


$$
x \in \Omega: c(T) \frac{\partial T(x, t)}{\partial t}=\lambda_{0} \nabla^{2} T(x, t)
$$

The essence of the method consists in the correction of the local temperature values found for transition $t^{f-1} \rightarrow t^{f}[1,3,4]$.

The interval $\left[T^{\infty}, T_{0}\right]$ (ambient temperature, initial temperature) is divided into sub-intervals for which one assumes the constant value of thermal capacity $C(T)$, this means $C(T)=c_{m}$, for $B_{m}<T<B_{m+1}$ (Figure 3). The computations are realized assuming that the entire domain considered corresponds conventionally to the basic phase (e.g. the 'hottest' phase) with parameter $c_{0}$. In this way the problem becomes the linear one. Next, the temperatures obtained for transition $t^{f-1} \rightarrow t^{f}$ in a homogenous area are corrected in a special way ( the procedure corresponding to the cooling process is discussed below, the procedure concerning the heating process is very similar).

Let us assume that the temperature at the point $x^{l}$ corresponds to phase $m$ Figure 3. We denote

$$
\begin{aligned}
& \Delta_{m}=c_{m}\left(T_{l}{ }^{f}-B_{m+1}\right) \\
& \Delta_{m+1}=\Delta_{m}+c_{m+1}\left(B_{m+1}-B_{m+2}\right) \\
& \Delta_{m+2}=\Delta_{m+1}+c_{m+2}\left(B_{m+2}-B_{m+3}\right)
\end{aligned}
$$

where: $B_{m}, B_{m+1}, B_{m+2}, \ldots, B_{M+1}$ are the border temperatures limiting the successive 'phases'. From the physical point of view the values $\Delta$ are the changes of unitary enthalpy $\left[\mathrm{J} / \mathrm{m}^{3}\right]$ corresponding to the changes of temperature shown in Figure 2.

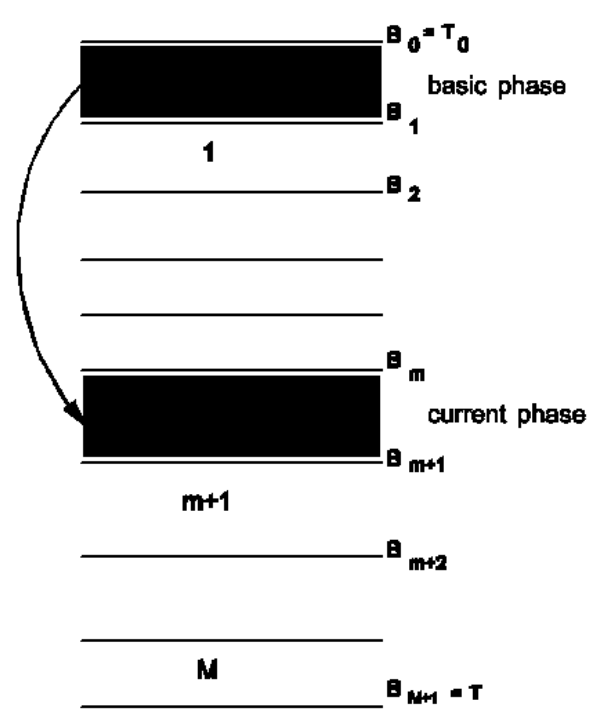

Fig. 3. The phases distinguished in the interval $\left[T^{\infty}, T_{0}\right]$ 
The procedure of temporary temperature correction method at the considered point $x^{l}$ is the following:

If

$$
c_{0}\left(T_{l}^{f-1}-T_{l}^{f}\right)<\Delta_{m}
$$

then the corrected value of $T_{l}^{f}$ results from the formula

$$
c_{0}\left({T_{l}}^{f-1}-T_{l}{ }^{f}\right)=c_{m}\left(T_{l}{ }^{f-1}-\hat{T}_{l}{ }^{f}\right)
$$

and next

$$
\hat{T}_{l}{ }^{f}=T_{l}{ }^{f-1}-\frac{c_{0}}{c_{m}}\left(T_{l}{ }^{f-1}-T_{l}{ }^{f}\right)
$$

In the case

$$
\Delta_{m}<c_{0}\left(T_{l}{ }^{f-1}-T_{l}{ }^{f}\right)<\Delta_{m+1}
$$

one obtains the following enthalpy balance

$$
c_{0}\left({T_{l}}^{f-1}-T_{l}^{f}\right)=\Delta_{m}+c_{m+1}\left(B_{m+1}-\hat{T}_{l}^{f}\right)
$$

and finally

$$
\hat{T}_{l}^{f}=B_{m+1}+\frac{\Delta_{m}}{c_{m+1}}-\frac{c_{0}}{c_{m+1}}\left(T_{l}{ }^{f-1}-T_{l}{ }^{f}\right)
$$

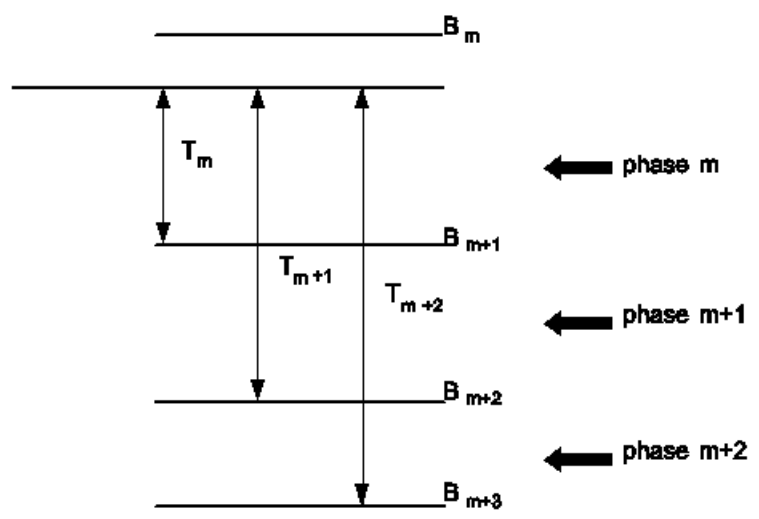

Fig. 4. Differences between $T_{i}^{f-1}$ and border temperatures

If

$$
\Delta_{m+1}<c_{0}\left(T_{l}{ }^{f-1}-T_{l}{ }^{f}\right)<\Delta_{m+2}
$$


then

$$
c_{0}\left(T_{l}{ }^{f-1}-T_{l}^{f}\right)=\Delta_{m+1}+c_{m+2}\left(B_{m+2}-\hat{T}_{l}^{f}\right)
$$

and

$$
\hat{T}_{l}^{f}=B_{m+2}+\frac{\Delta_{m+1}}{c_{m+2}}-\frac{c_{0}}{c_{m+2}}\left(T_{l}^{f-1}-T_{l}^{f}\right)
$$

The analogous formulas can be derived for the next transitions, but taking into account the exactness of the numerical solution, the big changes of temperatures are rather not accepted.

\section{Generalization of TFCM}

If one assumes that the thermal conductivity of material can be approximated in the form of piecewise constant function (similarly as volumetric specific heat Figure 3), this means $\lambda(T)=\lambda_{m}, B_{m}<T<B_{m+1}$, then the procedure presented in the previous chapter can be generalized in case of temperature-dependent $\lambda$. The computations are realized assuming that the entire domain considered corresponds conventionally to the basic phase (e.g. the 'hottest' phase) with parameters $c_{0}, \lambda_{0}$. So, the following energy equation is considered

$$
x \in \Omega: c_{0} \frac{\partial T(x, t)}{\partial t}=\lambda_{0} \nabla^{2} T(x, t)
$$

The similar equation for $m$ 'phase'

$$
c_{m} \frac{\partial T(x, t)}{\partial t}=\lambda_{m} \nabla^{2} T(x, t), \quad B_{m}<T<B_{m+1}
$$

can be rewritten as follows

$$
c_{m} \frac{\lambda_{0}}{\lambda_{m}} \frac{\partial T(x, t)}{\partial t}=\lambda_{0} \nabla^{2} T(x, t), \quad B_{m}<T<B_{m+1}
$$

or

$$
c_{m} \frac{\partial T(x, t)}{\partial t}=\lambda_{0} \nabla^{2} T(x, t), \quad B_{m}<T<B_{m+1}, \quad c_{m} \rightarrow c_{m} \frac{\lambda_{0}}{\lambda_{m}}
$$

In this way we 'return' to the problem described in the previous chapter. The volumetric specific heats corresponding to the successive 'phases' must be redefined in the proper way, of course. In a case of practical problems the Neumann condition is given in the form of boundary heat flux and then this condition should 
be rebuilt in a simple way. The same situation takes place when the Robin boundary condition is considered.

\section{Examples of computations}

The first example concerns the cooling of plate (1D problem, $L=0.05 \mathrm{~m}$ ). The input data for the basic problem are equal to $\lambda_{0}=1 \mathrm{~W} / \mathrm{mK}, c_{0}=10^{6} \mathrm{~J} / \mathrm{m}^{3} \mathrm{~K}$ while the rearranged solution corresponds to $\lambda_{m}=0.5$ and $c_{m}=2 \times 10^{6}$.

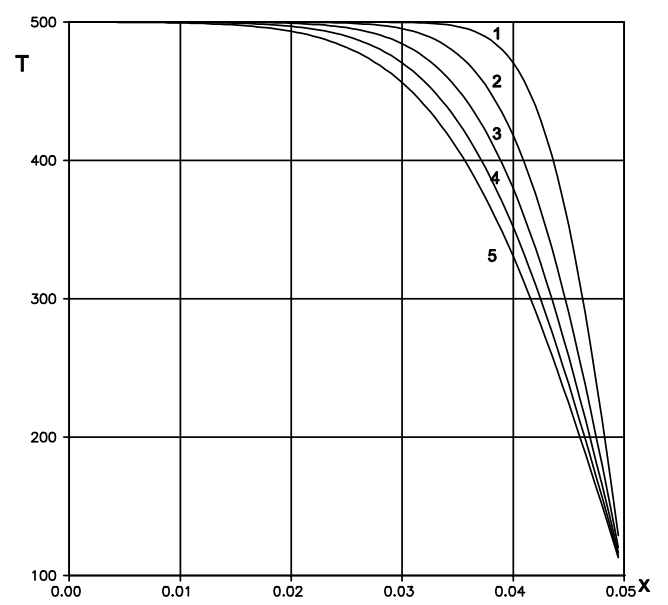

Fig. 5. Solution of the basic problem (Dirichlet conditions)

Additionally, the Dirichlet conditions are taken into account and the boundary temperatures are equal to 100 and $500^{\circ} \mathrm{C}$. Initial temperature equals $500^{\circ} \mathrm{C}$. The basic problem has been solved using the first scheme of the BEM for parabolic equations [3,4] and the temperature profiles for times 1, 2, 3, 4 and 5 minutes are shown in Figure 5.

Next, using the procedure described in the previous chapter, the solution obtained has been transformed in order to take into account the other values of thermophysical parameters. This solution is shown in Figure 6.

To check the effectiveness of procedure proposed the same problem has been solved directly for $\lambda_{m}=0.5$ and $c_{m}=2 \times 10^{6}$, the results are shown in Figure 7 .

One can see that the temperature profiles are practically the same.

The second example has been solved under the assumption that the boundary conditions correspond to the Neumann ones. In particular, the boundary heat fluxes are equal to 0 and $10000 \mathrm{~W} / \mathrm{m}^{2}$. The results of computations are shown in Figures 8, 9,10 and both the direct solution for new input data and the rearranged one are very close. 


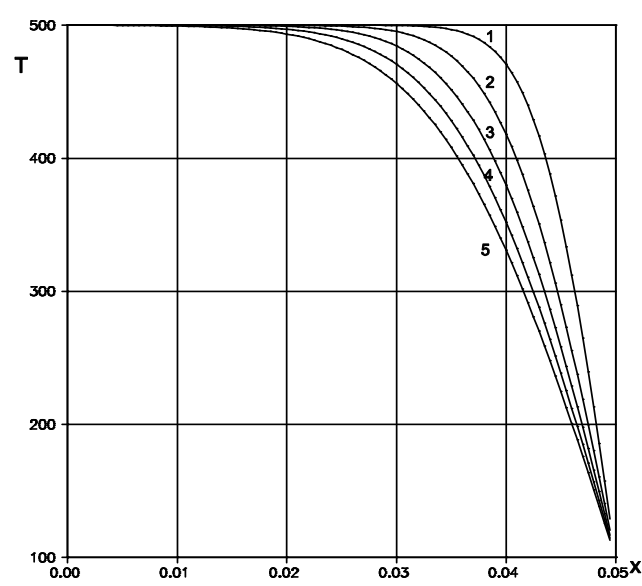

Fig. 6. Solution for new parameters

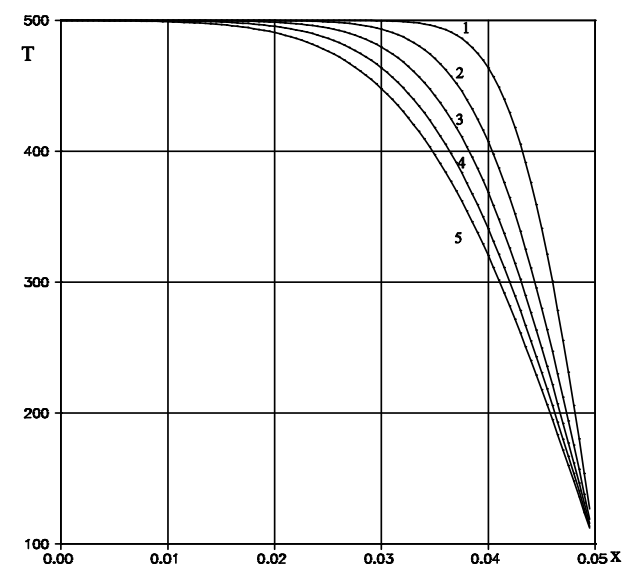

Fig. 7. The direct solution for new input data

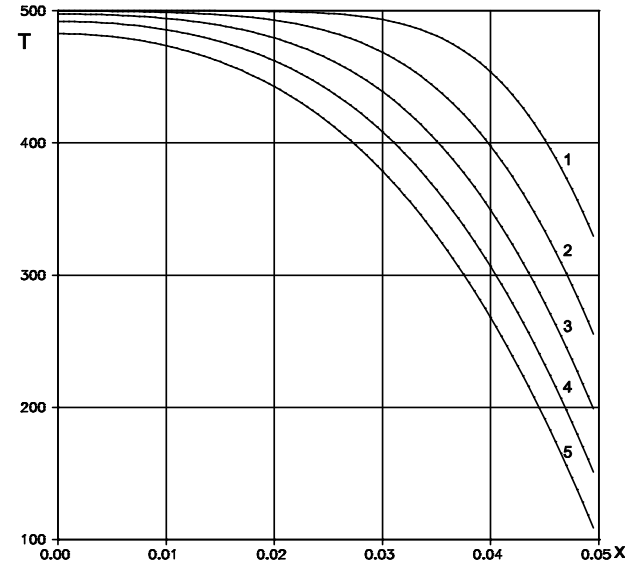

Fig. 8. The basic solution 

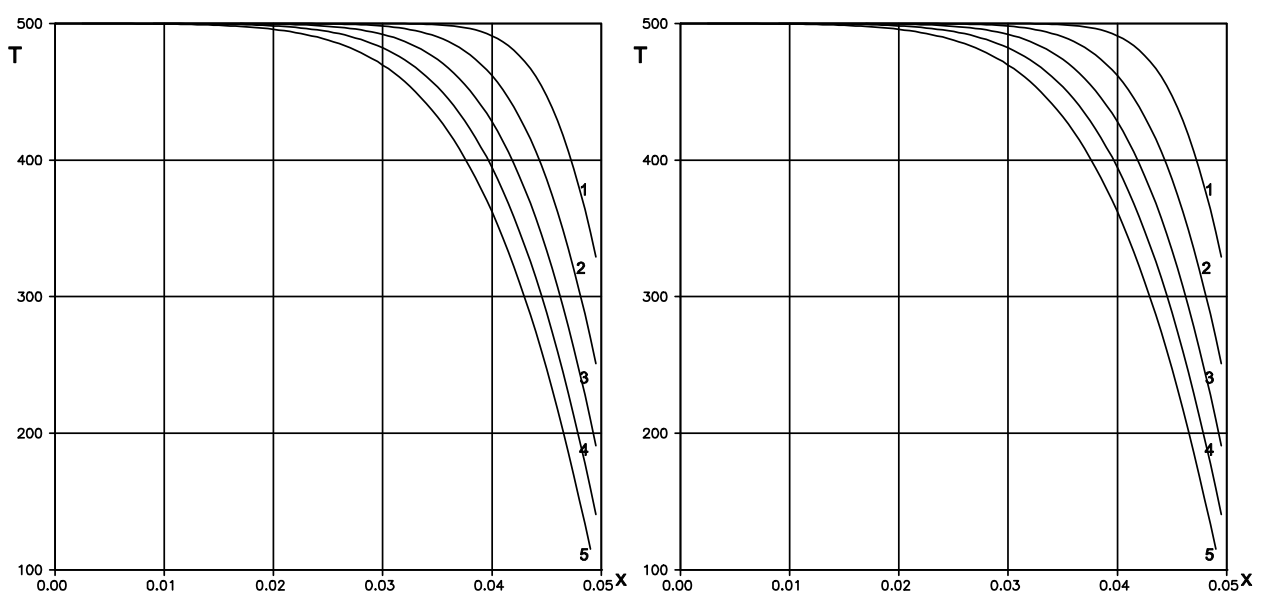

Fig. 9. The rearranged and direct solutions

The next example concerns the simulation of continuous casting technology (production of rectangular steel cast strands using the vertical plant). In particular, the thermal processes proceeding in the primary cooling zone are analyzed. The mathematical model of solidification is based on the method called 'a one domain approach' $[10,11]$ and then the evolution of latent heat is taken into account by the introduction of substitute thermal capacity $[12,13]$. This parameter can be assumed in the form of piecewise constant function. The same assumption can be accepted as an approximation of steel thermal conductivity. The energy equation describing the process is transformed to the form of the typical Fourier equation by the introduction of a moving co-ordinate system (wandering cross section method [14]). Let us assume that $T_{S}=1470{ }^{\circ} \mathrm{C}$ and $T_{L}=1505^{\circ} \mathrm{C}$ are the border temperatures corresponding to the transitions solid state - mushy zone - molten metal. Then the substitute thermal capacity of carbon steel $(0.44 \%$ C) is given as $c_{S}=4.875 \mathrm{MJ} / \mathrm{m}^{3} \mathrm{~K}$ (solid), $c_{M}=62.09 \mathrm{MJ} / \mathrm{m}^{3} \mathrm{~K}$ (mushy zone), $c_{L}=5.904 \mathrm{MJ} / \mathrm{m}^{3} \mathrm{~K}$ (molten metal), while $\lambda_{S}=35 \mathrm{~W} / \mathrm{mK}, \lambda_{M}=27.5 \mathrm{~W} / \mathrm{mK}, \lambda_{L}=20 \mathrm{~W} / \mathrm{mK}$. Pouring temperature $T_{p}=1550^{\circ} \mathrm{C}$. The dimensions of cast strand section are equal to $1 \times 0.2 \mathrm{~m}$. Pulling rate $w=0.02 \mathrm{~m} / \mathrm{s}$ (this information is necessary at the stage of energy equation formulation), boundary heat flux given on the lateral surface of cast strand $q=2 \times 10^{5} \mathrm{~W} / \mathrm{m}^{2}$, the length of primary cooling zone $L=0.8 \mathrm{~m}$. The computations have been realized for the molten metal parameters ('basic phase'). The problem (the wandering cross section method allows one to determine the temperature field in traverse sections of casting for different values of vertical coordinate) has been solved using the first scheme of boundary element method for 2D parabolic equations. In Figure 10 the cooling curves at the points located along the symmetry axis of a longer flank of the section are presented. The successive curves correspond to the $x=0.06,0.07,0.08,0.09,0.1 \mathrm{~m}$ (external boundary). 


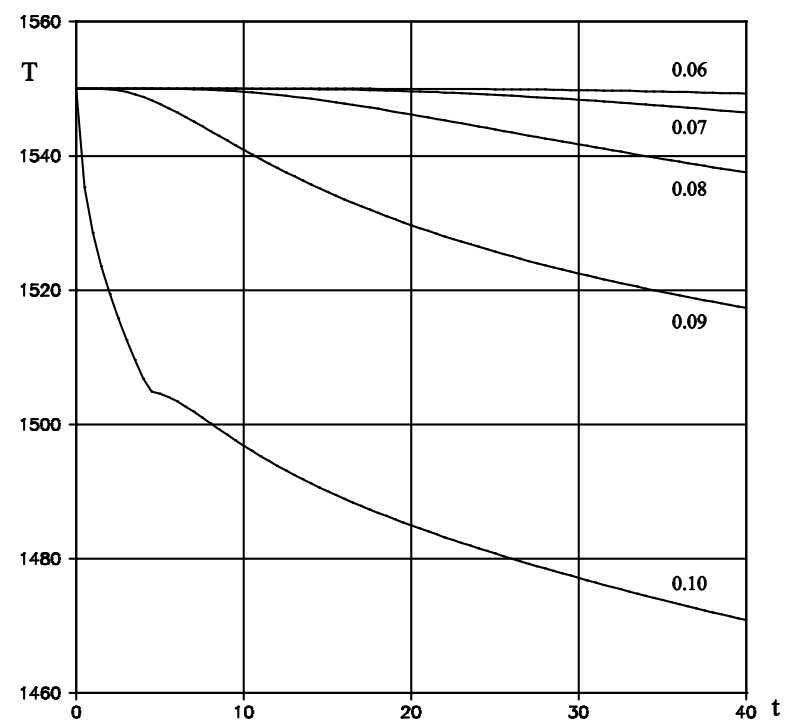

Fig. 10. Cooling curves

The solution obtained was compared with the solution found using the 'house in' FDM program simulating the non-linear and transient heat conduction process. The conformity of both solutions is quite satisfactory.

\section{Conclusions}

The different methods of artificial 'linearization' of boundary-initial problems connected with the heat conduction give the possibilities to transform (at the stage of numerical modeling) the task considered to the linear one whose approximate solution is essentially simpler. The most important applications of procedures presented appear in the case of boundary element method employment, but such an approach can be also used as the additional procedure complementary to the others methods of heat transfer modeling.

\section{References}

[1] Mochnacki B., Suchy J.S., Numerical methods in computations of foundry processes, PFTA, Cracow 1995.

[2] Hong C.P., Umeda T., Kimura Y., Numerical models for casting solidification problems, Metall. Trans. B, 1984, 15B, 101-105.

[3] Majchrzak E., Boundary element method in heat transfer problems, Publ. of Czestochowa University of Technology, Czestochowa 2001.

[4] Majchrzak E., Boundary element method in numerical modeling of solidification process, Progress of Foundry Theory and Practice, Polish Academy of Sciences, Foundry Commission, 2009. 
[5] Majchrzak E., Dziewonski M., Kaluza G., Application of the boundary element method coupled with the artificial source procedure for numerical modeling of freezing process, Scientific Research of the Institute of Mathematics and Computer Science 2009, 1(8), 105-112.

[6] Rogers J., Berger A., Ciment M., The alternating phase truncation method, for a Stefan problem, SIAM J. Num. Anal. 1979, 15, 562-587.

[7] Kapusta A., Mochnacki B., Analysis of heat transfer processes in cylindrical radial continuous casting volume, Bull. of the Pol. Ac. of Sc., Techn. Sciences, 35, 5-6 (1088) 309-320.

[8] Majchrzak E., Mendakiewicz J., Application of the generalized alternating phase truncation method for numerical modeling of cast iron solidification, Solidification of Metals and Alloys 1993, 18, 103-112.

[9] Mochnacki B., Szopa R., Generalization of temporary temperature field correction method in numerical modeling of solidification, Archives of Foundry Engineering 2008, 8, 1, 219-222.

[10] Majchrzak E., Mochnacki B., Identification of thermal properties of the system casting - mould, Materials Science Forum 2007, Vols. 539-543, 2491-2496.

[11] Mochnacki B., Computational simulations and applications. Numerical modeling of solidification process (Chapter 24), Ed. Jianping Zhu, INTECH, 2011.

[12] Mochnacki B., Majchrzak E., Numerical modeling of casting solidification using generalized finite difference method, Materials Science Forum 2010, Vols. 638-642, 2676-2681.

[13] Majchrzak E., Mochnacki B., Suchy J.S., Identification of substitute thermal capacity of solidifying alloy, Journal of Theoretical and Applied Mechanics 2008, 46, 2, 257-268.

[14] Majchrzak E., Mochnacki B., Identification of cooling conditions in domain of continuous casting mould, Proceedings of the International Conference on Advanced Materials and Processing Technologies, AMPT, Dublin 2003, 724-727. 
